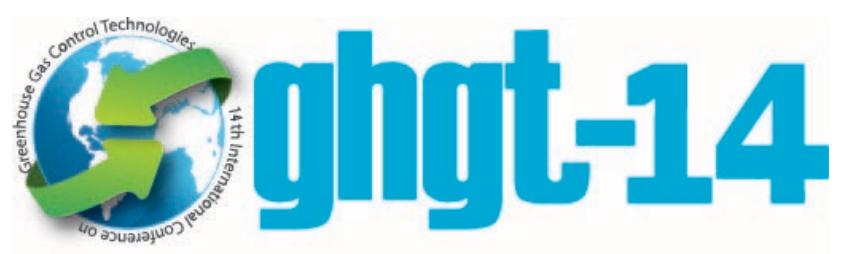

14th International Conference on Greenhouse Gas Control Technologies, GHGT-14

$21^{\text {st }}-25^{\text {th }}$ October 2018, Melbourne, Australia

\title{
CO2 storage site characterisation at Harvey, Western Australia using non-conventional seismic survey geometries
}

\author{
Milovan Urosevic ${ }^{\mathrm{a}, *}$, Sasha Ziramov ${ }^{\mathrm{a}}$, Roman Pevzner ${ }^{\mathrm{a}}$, Konstantin Tertyshnikov ${ }^{\mathrm{a}}$, Sinem \\ Yavuz $^{\mathrm{a}}$, Anton Egorov ${ }^{\mathrm{a}}$, Andrej Bona ${ }^{\mathrm{a}}$, Valeriya Shulakova ${ }^{\mathrm{c}}$, and Dominique Van Gent ${ }^{\mathrm{b}}$ \\ ${ }^{a}$ Curtin University, 26 Dick Perry Avenue, Kensington 6151, Western Australia
}

${ }^{b}$ Department of Mines, Industry Regulation and Safety, 6230 Banbury, Australia

${ }^{c}$ CSIRO, 26 Dick Perry Avenue, Kensington 6151, Western Australia

\begin{abstract}
This stage of the South West Hub Carbon Capture and Storage (CCS) project aims to produce an in-depth integrated study of the fault and fracture network surrounding the Harvey 3 well to obtain an understanding of their propensity to act as conduits for the injected $\mathrm{CO}_{2}$. An additional interest to the seismic component of the study was to find an optimum $\mathrm{CO}_{2}$ sequestration monitoring methodology. This was of key importance since acceptance by the farming community across the area of the potential reservoir extent is crucial for successful completion of the project. These two factors were taken into account for the survey design that was comprised of more conventional components consisting of simultaneous recorded 3D surface and 3D VSP data sets and an alternative component that recorded multi-offset VSP (MOVSP) survey along the public roads. A comparative analysis of seismic imaging achieved with different geometries is discussed. One of the important results was that MOVSP surveys produced good results and hence this technique may be preferred for monitoring $\mathrm{CO}_{2}$ sequestration in this area. This was since it caused no disturbance to the farming ground and it was much more likely to gain acceptance with the landowners.

Keywords: 3D seismic surveys, MOVSP, $\mathrm{CO}_{2}$ squestration monitoring
\end{abstract}

\section{Introduction}

The South West Hub Carbon Capture and Storage project is a leading initiative to reduce carbon dioxide emissions in Western Australia. It is a staged project that involves collecting and analysing data and samples from the Lesueur Sandstone formation to test its feasibility as a $\mathrm{CO}_{2}$ reservoir. The Government of Western Australia, Department of Mines, Industry Regulation and Safety face the challenge of proving containment security for the SW $\mathrm{Hub}$ and the local community is expecting a sound scientific evaluation of containment potential for $\mathrm{CO}_{2}$. Characterization of this reservoir and its related structures are critical to the project that is largely based on the residual trapping mechanism. Hence, in 2014 a large 3D seismic survey covering $110 \mathrm{~km}^{2}$ of the potential storage

\footnotetext{
* Milovan Urosevic. Tel: +61 89266 2296; fax: +61 892663407.

E-mail address: M.Urosevic@curtin.edu.au
} 
area was acquired for the SW Hub project acquired. The survey parameters were suited for imaging deeper structures below $300 \mathrm{~m}$ depth. The 3D survey was not meant to present a base-line survey, rather help with placing the stratigraphic boreholes Harvey 2, 3 and 4.

Harvey $3(\mathrm{H} 3)$ well was drilled in a highly prospective zone for $\mathrm{CO}_{2}$ geo-sequestration found west of the major dislocation, the so-called F10 fault, but it remained poorly covered with seismic due to access restriction at the time. In 2016, a large fire swept through the area surrounding the H3 well. Unfortunately, it created an opportunity for additional seismic investigations previously not permitted in the area. However, the numerous restrictions have stayed in place, which forced us to come up with a fairly non-standard survey design. This experimental survey had multiple objectives concerning the future $\mathrm{CO}_{2}$ sequestration program. The primary objective was the structural characterisation of the area surrounding the $\mathrm{H} 3$ well. The second objective involved seismo-stratigraphic studies including quantitative analysis of the strata overlying prospective reservoir. Finally, we aimed to establish an optimum $\mathrm{CO}_{2}$ sequestration methodology, suitable for an area with highly restrictive site access.

\section{Seismic Data Acquisition}

The designed survey consisted of simultaneous borehole and surface recordings combined in an unconventional way (Fig. 1). The main component of the survey was the borehole study that involved 550 multi-offset Vertical Seismic Profiling (MOVSP) vibrating positions. By products included; Zero-offset VSP, 2D seismic lines, and a small 3D surface seismic survey that shared the same source points with the 3D VSP survey (Fig. 1). Ten threecomponent (3C) slim-wave shuttles (Sercel), positioned every $15 \mathrm{~m}$ were used to cover the entire length of the H3 well for MOVSP survey. A 3D VSP survey was recorded at one of the MOVSP depth range of 900-1035 m, some $500 \mathrm{~m}$ above the top of the reservoir. It utilised 350 additional shots (14 source lines) fired for 3D surface seismic in the W-E direction.

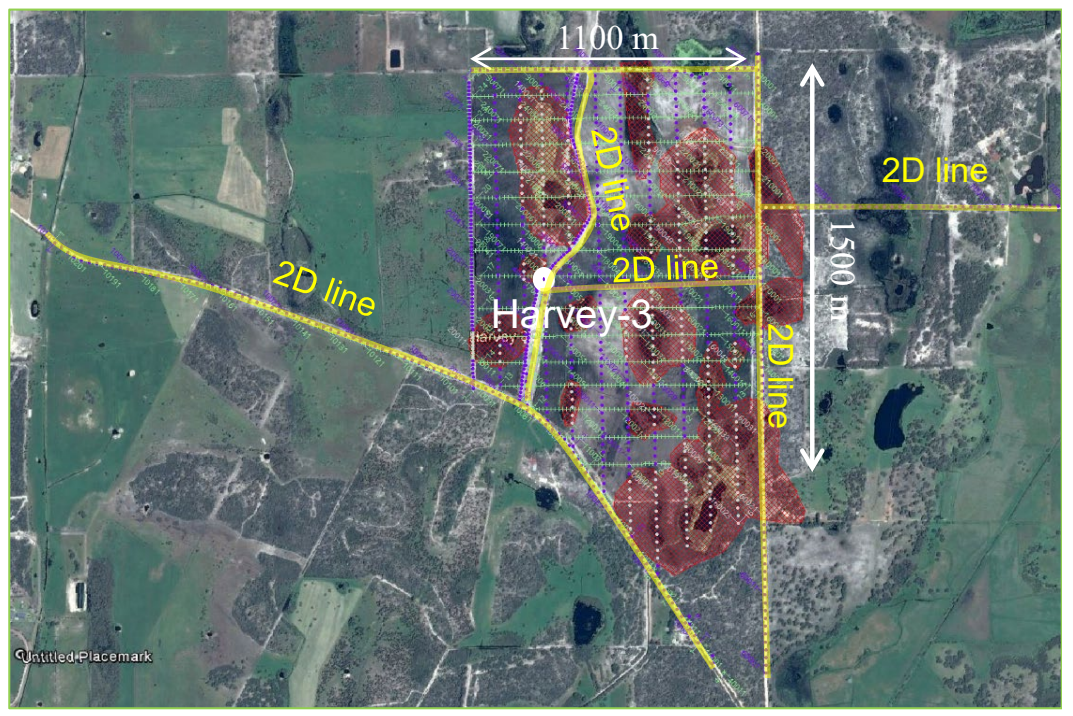

Fig. 1. H3 borehole and surface seismic survey geometry. Yellow solid lines denote public roads. Orthogonal dashed lines stand for source lines (south-north) and receiver lines (west-east).

For each depth level, seismic energy was generated by two vibroseis trucks (UniVib) shooting in a flip-flop manner at 550 surface locations, making a total of 5,500 OVSP points. MOVSP points were distributed along the dirt public roads, where permission was always expected to be granted, as this was most likely scenario for any future $\mathrm{CO}_{2}$ monitoring methodology. Sweep parameters were $6-150 \mathrm{~Hz}, 24$ seconds, single sweep/VP. An additional $1000 \mathrm{VP}$ points were to be used for 3D VSP and 3D surface seismic. A crew of eight people (staff and PhD students) acquired this comprehensive dataset in only 11 days. Combining MOVSP points with a 3D grid produced 
an extended but irregular fold map (Fig. 2). This irregular fold map required specific processing steps to reduce the footprint. The acquired data set was processed with the following geometries:
A. 3D surface seismic cube
B. $4 \times 2 \mathrm{D}$ seismic lines
C. 3D VSP cube
D. ZVSP, OVSPs
E. MOVSP involving roads only
F. MOVSP involving all shots $(3 \mathrm{D}+$ roads $)$.

The acquisition of this complex data set was carried out in two stages (Urosevic et al., 2017). The idea was to optimise the acquisition to complete it in the shortest possible time to avoid any negative reactions from the farming community. The first stage of acquisition consisted of MOVSP surveys. A Sercel Slim-Wave VSP tool with ten component shuttles was deployed in the H-3 well. The survey was conducted with eight to nine shuttles (Table 1). For each depth level, 451 VPs were distributed along dirt roads (Fig. 1). The same VPs were repeated for the next depth level. Hence, a total of 4510 VPs were used for the MOVSP survey. For one depth level occupied, we shot public roads plus a 3D source-receiver grid, producing simultaneously a 3D surface reflection cube and a 3D VSP data set, as is shown in table 1 . The second stage of the acquisition utilised 1280 shots. Circa 829 VPs were fired along the 3D source grid to simultaneously acquire 3D surface and VSP data sets. In this way, we had 451 OVSP surveys, each with the ability to produce an independent depth image in the plane connecting each VP to the borehole. Alternatively, we can use all 4510 points, which would be equivalent to a 3D VSP survey that utilised a continuous geophone array along the entire borehole trajectory.

Table 1. MOVSP depth levels in order of execution. For each depth level, 451 VP points were spread along dirt roads (Fig. 1). The total number of MOVSP shots is 4510. 3D VSP data acquisition was executed last.

\begin{tabular}{|c|c|c|c|l|}
\hline Stage & VSP depth level $(\mathrm{m})$ & No. of shuttles & No. of shots & Comments \\
\hline \multirow{4}{*}{ I } & $290-395$ & 8 & 451 & Public roads (PR) \\
\cline { 2 - 5 } & $1340-1445$ & 8 & 451 & PR \\
\cline { 2 - 5 } & $1235-1340$ & 8 & 451 & PR \\
\cline { 2 - 5 } & $1115-1235$ & 9 & 451 & PR \\
\cline { 2 - 5 } & $980-1100$ & 9 & 451 & PR \\
\cline { 2 - 5 } & $845-965$ & 9 & 451 & PR \\
\cline { 2 - 5 } & $740-845$ & 8 & 451 & PR \\
\cline { 2 - 5 } & $620-725$ & 8 & 451 & PR \\
\cline { 2 - 5 } & $380-485$ & 8 & 451 & PR \\
\hline II & $500-605$ & 8 & 1280 & 3D grid + PR \\
\hline
\end{tabular}


Table 2. Acquisition parameters for each survey stage. Note that some surface lines utilized $3 \mathrm{C}$ receivers. This will enable us to produce an expanded image around the H-3 well in all three directions (X, Y and Z).

\begin{tabular}{|l|l|l|l|}
\hline \multicolumn{2}{|c|}{ Stage I } & \multicolumn{2}{c|}{ Stage II } \\
\hline Number of Source Line & 6 & Number of Source Line: & 15 \\
\hline Number of Sources & 451 per VSP level & Number of Sources: & 829 \\
\hline Source Spacing (m) & 15 & Source Spacing (m) & $30 \mathrm{~m}$ \\
\hline Source Line spacing (m) & 440 & Source Line spacing (m) & $260 \mathrm{~m}$ \\
\hline Total Source Line Length (km) & $9.1 \mathrm{~km}$ & S/R Line Lengths (km) & $23.45 \& 22.27$ \\
\hline Number of Receiver Line & $1(\mathrm{H}-3$ well) & Number of Receiver Line: & 14 \\
\hline Number of VSP levels & 10 & Receiver Lines 9-14 & $3723 \mathrm{C}$ geophones \\
\hline Number of Receivers & 78 depth locations & Number of receivers & 1503 \\
\hline Receiver Spacing (m) & 15 & Receiver Spacing (m) & $15 \mathrm{~m}$ \\
\hline No of shuttles & $8-9$ & Receiver Line spacing (m) & $150 \mathrm{~m}$ \\
\hline Depth coverage & $290-1445 \mathrm{~m}$ & Borehole depth level (m) & $500-605$ \\
\hline Source 26,000 Lb Vibroseis: Single sweep: 6-150Hz, Linear over 24 seconds & & \\
\hline
\end{tabular}

Furthermore, the total number of 4510 VPs may be decimated in several stages to infer the minimum amount of MOVSP points required to produce an acceptable quality depth image around the borehole. Such optimisation is potentially of high importance for future surveys as it may result in substantial cost savings. Finally, the MOVSP images (full set and divided) can be compared to 3D VSP images to further refine or devise novel approaches to borehole-based characterisation and/or $\mathrm{CO}_{2}$ monitoring methodologies.

\section{Data processing and analysis}

Initial processing involved generating a first time-depth-velocity relationship from the zero-offset VSP data (shot closest to the borehole). The next step involved binning of the full data set, which included all shot-receiver positions for 3D surface reflection processing (Fig. 2). Clearly different data subsets are easily derived from here; 2D lines, 3D involving orthogonal design only and all data as shown in Fig. 2. For the purpose of structural characterisation, we processed the full surface data set. In the first instance, a conventional data flow was only to be refined in the second step through the utilisation of pre-stack time migration (PSTM). ZVSP corridor stack and offset VSP (OVSP) data mapped to two-way time correlated well with surface seismic (Fig. 3). ZVSP derived velocity function was used as a guide function for the interactive velocity analysis. This analysis was subsequently refined through an iterative time and depth image gather analysis. Selected in-lines from the PSTM processed cube are shown in Fig. 4. Preserved relative amplitudes processing enabled subsequent qualitative and quantitative analysis from the data cube.

Subsequent data processing and analysis involved 3D VSP and MOVSP sets. To understand this stage shot lines were divided into two groups: (a) lines 1-6 that involved vibrator truck driving along roads of opportunity (roads with public access), and (b) Lines 7-15 that were distributed across several farm paddocks and comprised shot line grid for 3D surface and 3D VSP surveys (Fig. 5). Set (a) involved 451 vibrators positions (VPs) that were reshot 10 times for 10 consecutive borehole receiver array depth positions. VSP imaging involved these two sets (MOVSP and 3D VSP) including various decimations in a search for the optimum imaging and thus monitoring methodology that would involve borehole receivers and VPs placed along the tracks of opportunity. 


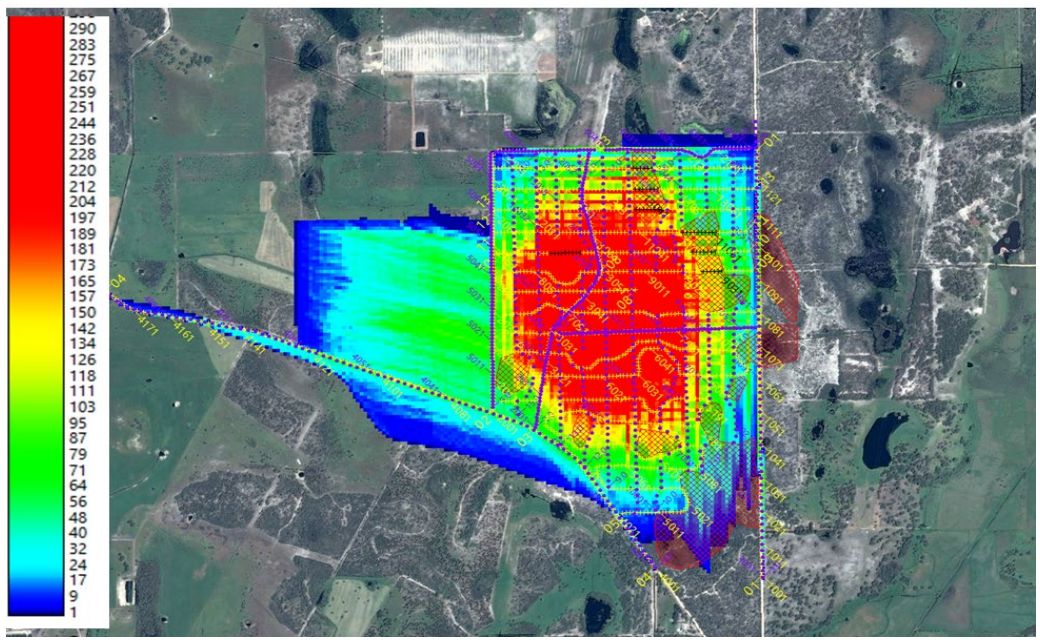

Fig. 2. Surface fold map involving all shot points. Maximum fold approaches 300 in the region surrounding H-3 well.

Evaluation of the results was made through a comparative study involving surface 3D and various VSP data sets. One of the in-house developed imaging algorithms involved a Kirchhoff VSP depth migration that inputted data of arbitrary geometry and outputted it into any selected plane. This is shown in Fig. 6 where all the "road line" MOVSP data were used to create and image along a single west-east line. Such redundancy of data also allowed us to select only shots with the highest signal to noise ratio (SNR) to create a high quality depth image. This approach gives us significant flexibility for creation of seismic images from VSP data in any particular direction that is of interest to reservoir characterisation and monitoring.

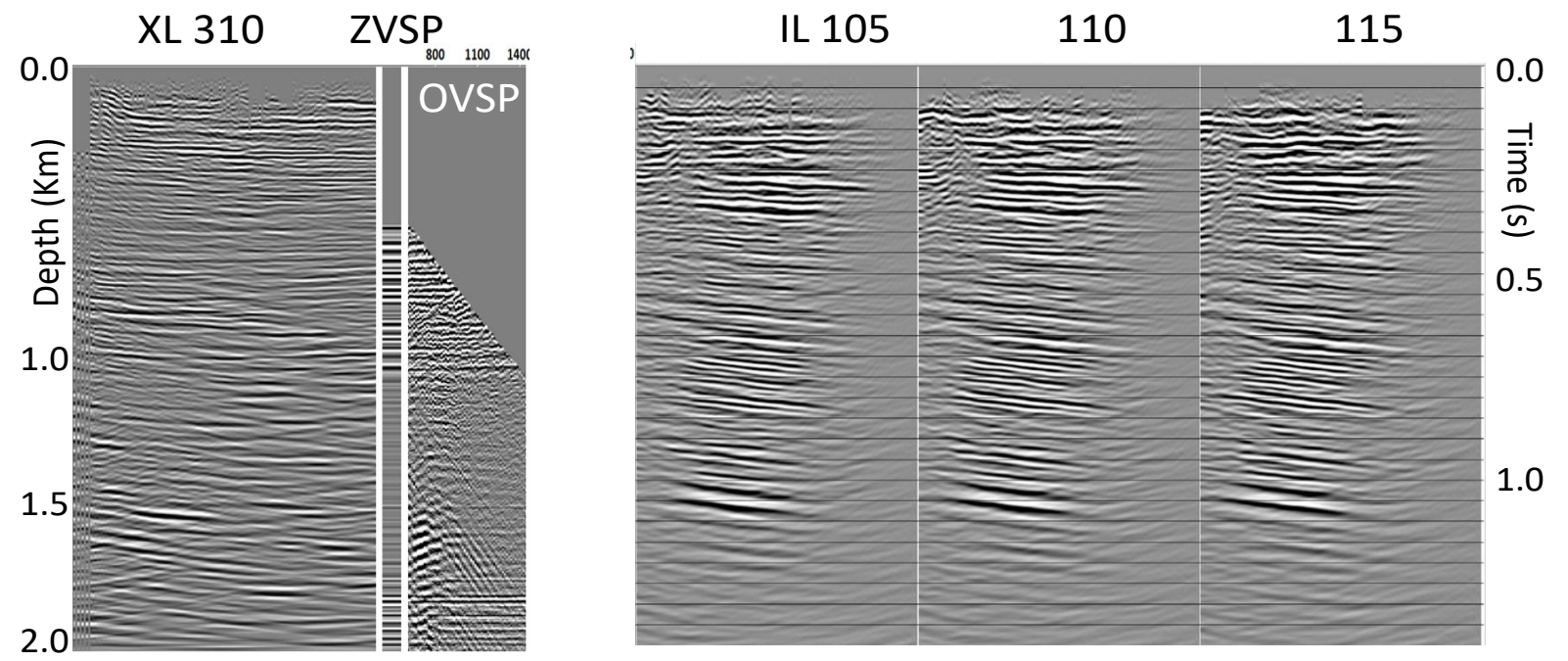

Fig.3. Left is XL 310, middle is VSP corridor stack and right is two-way short-offset VSP.

Fig. 4. Selected in-lines from PSTM seismic cube. 


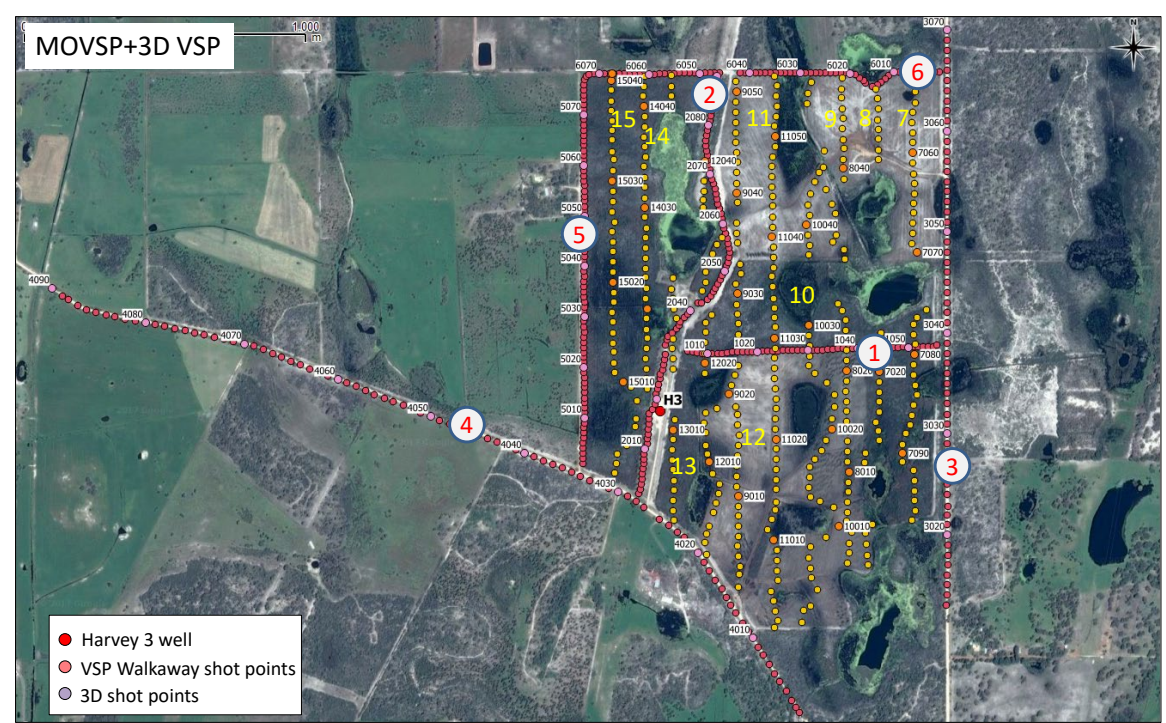

Fig.5. Disposition of source lines within the survey. Lines 1-6 were shot along the public roads. Lines 7-15 were within a 3D volume shot on virgin ground.

The image quality of different VSP data sets was initially evaluated against the PSTM seismic cube, requiring reconversion of the VSP images from depth to time. An example of this is shown in Fig. 7 where a 3D VSP image formed by utilising all shot lines (1-15) for image formation is displayed together with an appropriate in line extracted from the PSTM cube. In the high fold zone, VSP image compares well with surface. In the direction of line 4 (Fig. 5) the fold is very low but event continuity can be seen. This is true amplitude processing which causes uneven amplitudes as a product of different scaling by data fold. Figure 8 shows a very interesting and encouraging VSP imaging test. There we compare the MOVSP image along public roads with the full MOVSPS data set and 3D VSP. Clear images obtained by using only shots along public roads produced good and comparable results for the full data set image.

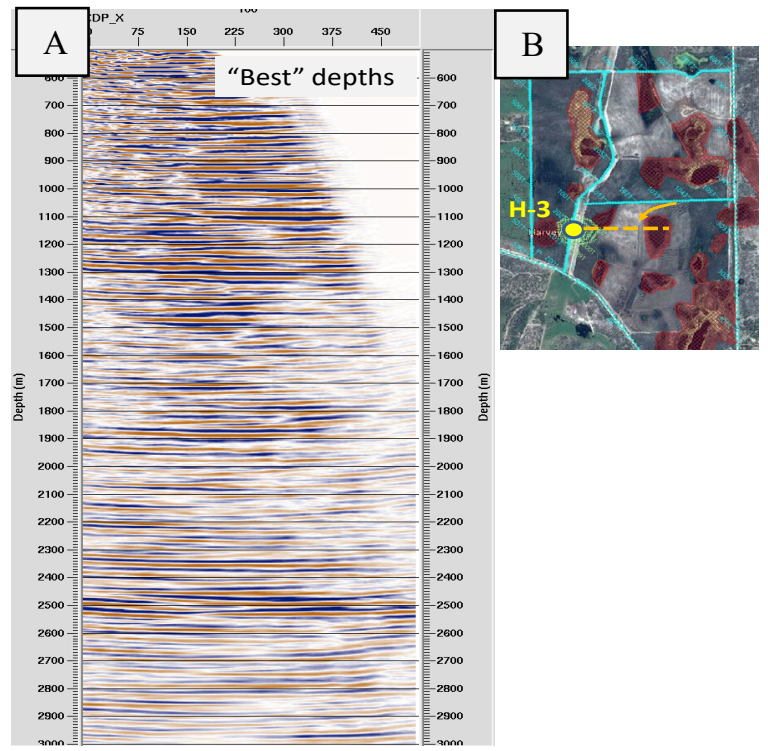

Fig.6. Data recorded along all roads (road lines) were migrated into a single W-E line (orange). Very good quality was enhanced by

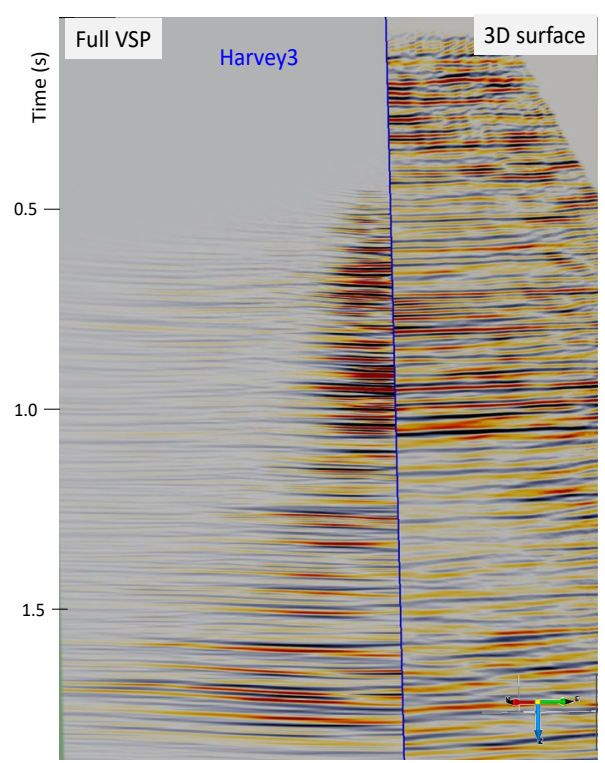

Fig. 7. In-line from 3D VSP cube obtained with full data data set (left) and 3D surface in-line (right). 
selection of the "best" depth levels (highest SNR).

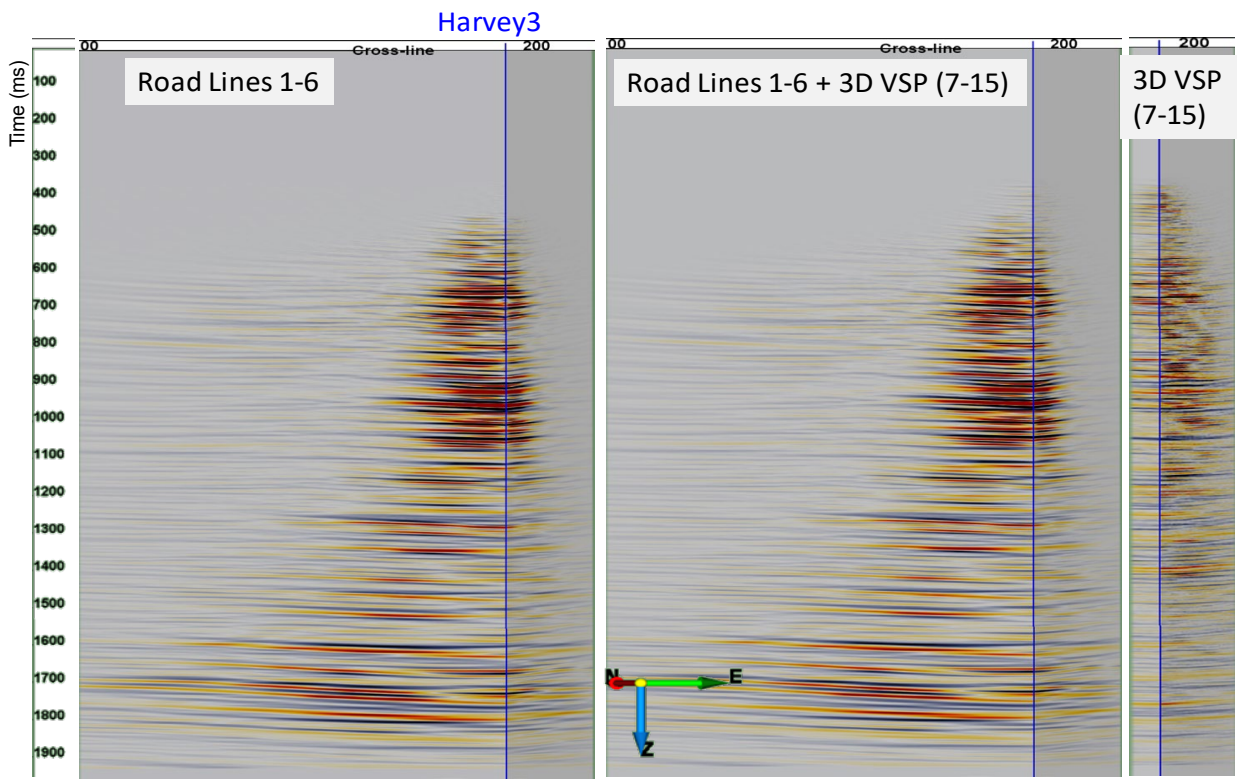

Fig. 8. VSP depth images obtained from: Shots along lines 1-6 (left), entire data set that is shots along lines 1-15 (middle) and 3D VSP obtained with shots along lines 7-15 (right). Note that first two panels are nearly identical so that contribution from line 7-15 is small.

The final investigations were aimed at testing the applicability of Full Waveform Inversion (FWI) of VSP data for rock characterisation. The main reason for looking into the VSP FWI approach was because borehole recording was generally had a greater SNR in comparison to surface recording and provided a very good starting model for the inversion. An additional and very important aim (of this project?) was to evaluate the feasibility of using single offset VSP for reservoir characterisation through inversion. This was an extreme but not impossible case that simulated very limited accessibility for either reservoir characterisation or follow up monitoring. The results of the FWI test (full elastic case) conducted with single offset shot VSP data are displayed in Fig. 9. Simple input models for $\mathrm{Vp}, \mathrm{Vs}$ and $\rho$ produced reasonable inversion results (Figure 9). This is very promising result which could pay the way for a new approach to seismic monitoring of $\mathrm{CO}_{2}$ sequestration at Harvey, WA.
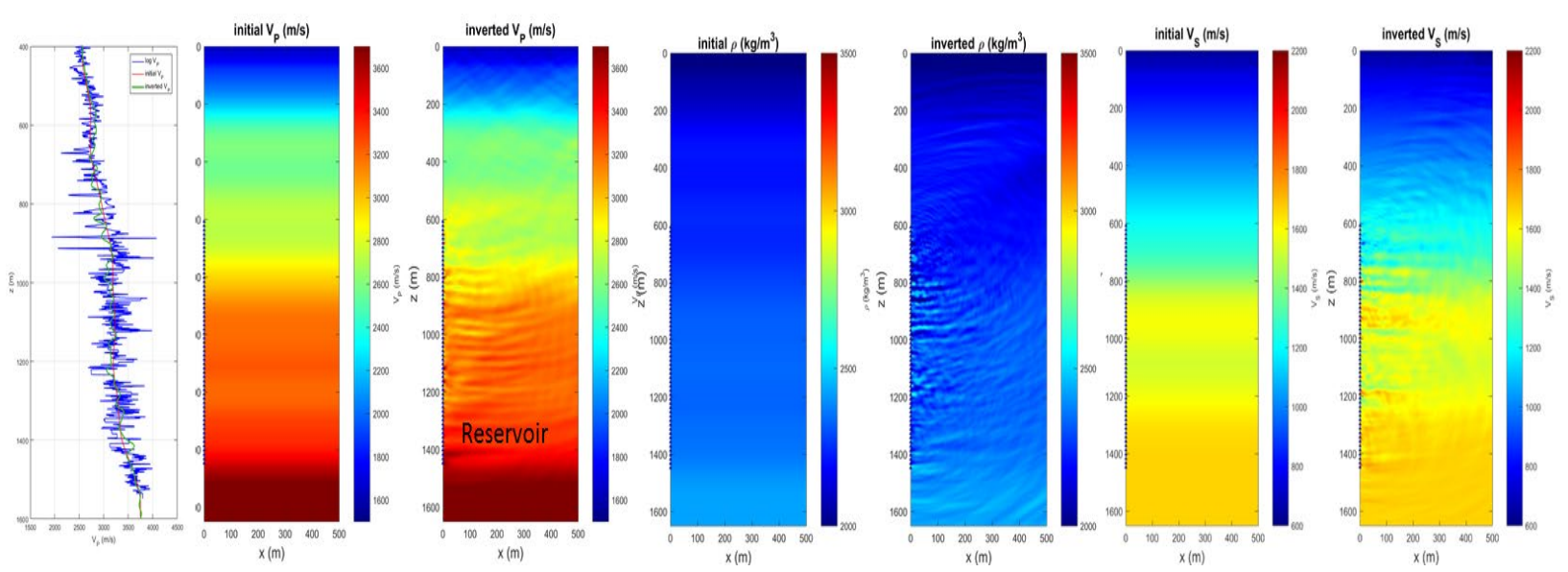
Fig.9. FWI of OVSP data. Log data (blue) and inverted Vp (red) are shown on the left, followed by: Intitial Vp mode and inverted Vp, initial density and inverted density and initial Vs model and inverted Vs.

\section{Conclusion}

The results obtained with the 3D surface, 3D VSP and individual OVSPs are quite exciting with respect to looking into alternative, zero footprint, site characterisation and subsequent $\mathrm{CO}_{2}$ sequestration monitoring. MOVSP surveys offer high flexibility for depth imaging and shows high potential for devising an alternative monitoring program that is likely to be accepted by the wider community. The quality of individual OVSP shots enabled us to run initial Full Waveform Inversion (FWI) tests. The high-quality inversion results obtained were essential for the lithological characterisation of reservoir formation, which was an unconventional sealing unit of particular importance to the sequestration project and this study. A new monitoring methodology that utilises opportunistic MOVSP surveys combined with the application of FWI may be the only possible way to precede with $\mathrm{CO}_{2}$ capture and sequestration projects in environmentally sensitive areas.

\section{Acknowledgements}

The authors wish to acknowledge financial assistance provided through Australian National Low Emissions Coal Research and Development (ANLEC R\&D). ANLEC R\&D is supported by Australian Coal Association Low Emissions Technology Limited and the Australian Government through the Clean Energy Initiative. We are grateful to Haliburton Company for donation of SeisSpace PROMAX processing package. We are also grateful to DECO geophysical company for the donation of RadExPro software that was largely used for VSP imaging.

\section{References}

[1] Urosevic, M., Ziramov, S., Pevzner, R., Tertyshnikov R., Popik, D., and Van Gent, D., 2017, CO2 storage site characterisation at the location of Harvey-3 well, Harvey, Western Australia: $2^{\text {nd }}$ EAGE/SEG Research Workshop 2017, 28-31 August 2017, Trondheim, Norway. 